Sādhanā Vol. 39, Part 4, August 2014, pp. 939-956. (C) Indian Academy of Sciences

\title{
Secret data embedding scheme modifying the frequency of occurrence of image brightness values
}

\author{
YILDIRAY YALMAN* and ISMAIL ERTURK \\ Computer Engineering Department, Turgut Ozal University, Ankara, Turkey \\ e-mail: yyalman@turgutozal.edu.tr; ierturk@turgutozal.edu.tr \\ MS received 5 April 2013; revised 19 June 2013; accepted 12 August 2013
}

\begin{abstract}
The main purpose of this presented work is to develop a data embedding method based on a new digital image histogram modification approach. The proposed scheme fundamentally is concerned about the frequency of occurrence of the image brightness values of the cover image for the data embedding procedures. The proposed scheme effectively realizes both perceptual invisibility and statistical invisibility so that obtained covered images are highly robust against common perceptual and statistical steganalysis techniques. The scheme provides reasonably higher payload values than its counterparts, as well as providing comparatively improved PSNR results.
\end{abstract}

Keywords. Image histogram; information security; data hiding; watermarking.

\section{Introduction}

Particularly in developed western societies, serious steps are taken in order to become an information society and many studies are made in this direction. In this context, individuals, institutions and organizations carry out their transactions electronically. Among those, tax, bill and fine payments, performing money transfers, shopping and reaching all the documents and information by using a personal computer or cell phone may be ranked. As a natural consequence of this situation, information security (IS) concepts have gained great importance in many areas such as banking, e-commerce, e-signature, distance learning, e-government applications and personal communications. The fact that individuals or states could not become effective separately in an information society this has become a current issue. As a part of the interdependence principle, an efficacious security approach will be inevitable by means of providing the security of all individuals, organizations and countries (Bensghir \& Altinsoy 2008).

Information-centric security approaches are generally addressed in two main topics such as personal and corporate IS. Serious increase in security breaches in the IS field diverts individuals and institutions to develop new safety measures by taking into account the contemporary software, hardware and physical solutions. At this point, a single safety measure to ensure the IS such as network security or software security is regarded as very insufficient although it is

${ }^{*}$ For correspondence 
considered important. For this reason, in order to ensure an effective IS, all requirements in this respect should be met with a lump sum point of view, by taking measures such as the security of hardware, software, network and communication, emission (tempest), staff, crypto, physical space, document, etc. (Sevgi 2001). Having carried out a simple literature review, it can be easily stated that one of the most important IS techniques is secret data embedding schemes or steganographic methods. In this context, based on a digital cover image, a new secret data embedding method is comprehensively presented in this study.

A wide variety of mathematical algorithms for data hiding have been implemented as computer programs for a decade. There has recently been enormous progress due to new development in hardware and software technologies in this field. Since the beginning of the 1950s, many data hiding techniques have been developed and implemented (Cox \& Miller 2008; Ni et al 2004; Yalman \& Erturk 2009). Nevertheless, the desired high level of information security has not been achieved in many areas. Consequently, both novel IS schemes and their optimization have often received a growing attention in conjunction with the new tools and methods (Yalman \& Erturk 2009).

In general, the well-known Least Significant Bits (LSB) approach for steganographic techniques refers to alteration of image pixels' LSBs for personal IS. In this study, the presented data hiding algorithm fundamentally utilizes the classical LSB data hiding on the Frequency of Occurrence $(\mathrm{FoO})$ of image Brightness Values $\left(\mathrm{V}_{\mathrm{B}}\right)$ (i.e., digital image histogram modifying). The obtained covered images are rationally robust against main steganalysis operations and provide high capacity and improved Peak Signal to Noise Ratios (PSNR). It has been well proved to be promising using data embedding schemes for authentication, fingerprinting, data security and other purposes. Third parties sometimes intervene to do unauthorised operations/attacks and might also unconsciously disrupt an embedded file or bits) in a covered multimedia in such data embedding implementations.

The most well-known and easiest of these operations are cropping, LSB changing and lossy compression. Moreover, geometrical operations on especially watermarking applications (such as warping, rotation and scattered tiles) have just come out, generally changing the pixels' locations of a covered/stego image (Yalman 2010). As a secondary contribution of this research paper, the proposed new scheme simply based on histogram modification is also fully usable for watermarking applications due to its inherent resistance to the basic geometric operations.

Essential points of the image processing and data embedding are clarified in the next section. The proposed new data embedding scheme and its details are introduced in the third section. Its evaluation, example applications and application results compared to those of the contemporary counterparts are given in the fourth section. Steganalysis evaluations and final comments are presented in the fifth and sixth sections, respectively.

\section{The digital image and data embedding fundamentals}

Basically, a fundamental picture element called as 'pixel' is the smallest discrete component of a digital image that is composed of a sequence of ' $n$ ' rows and ' $m$ ' columns (figure 1) (Cetin $\&$ Ozcerit 2009). The amount of bits representing a single pixel determines the colours that can be depicted. For instance, in the 24-bit colour standard, a colour monitor uses 24 bits for each picture element, allowing displaying $2^{24}(16,777,216)$ different colours.

Experimental applications show that, differences or deteriorations in pixel values cannot be readily sensed by the Human Vision System (HVS) (figure 2). This fact, also main motivation of this study, encourages the secret data embedding processes/techniques and similar applications 


\section{Columns}

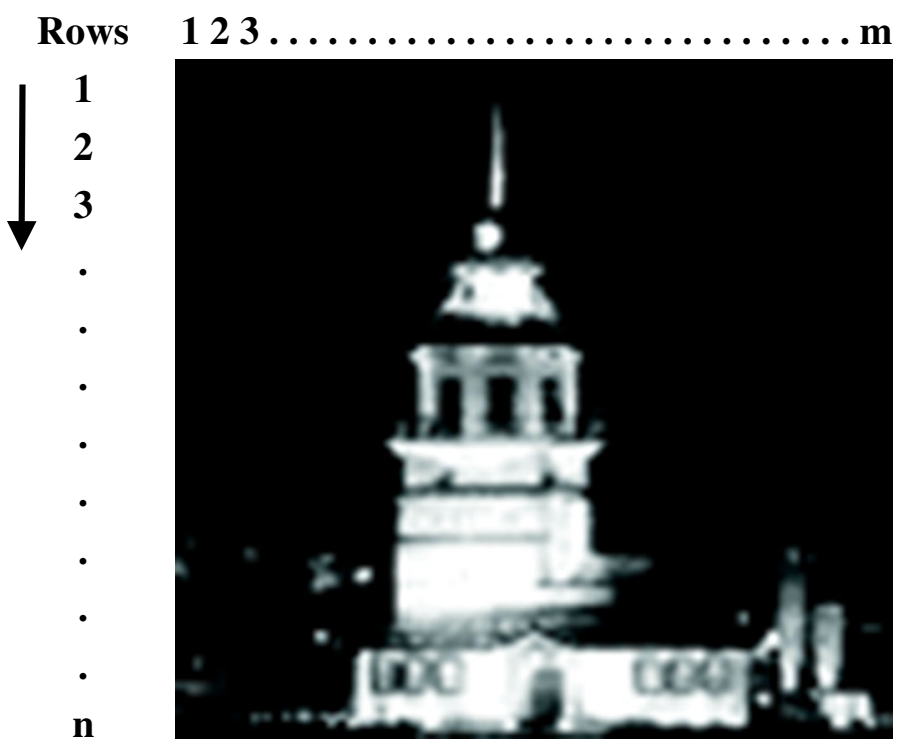

Figure 1. Representation of an image.

to utilise this very natural attribute. In these type of applications, a digital cover file (e.g., digital audio, image, video, etc.) is always required.

Steganography is generally described as a discipline of information hiding that anyone except from the coder and intended receiver can even understand that there is an embedded data/file (figure 3) (Chang \& Lin 2006; Huang \& Fang 2008; Chen \& Chang 2008; Hernandez-Castro et al 2006; Yalman \& Erturk 2009). Similarly, nowadays this discipline states disguise of digital information/data within computer files. On the other hand, cryptographic applications obscure the content as well as meaning of a secret data through encryption algorithms, though incapable
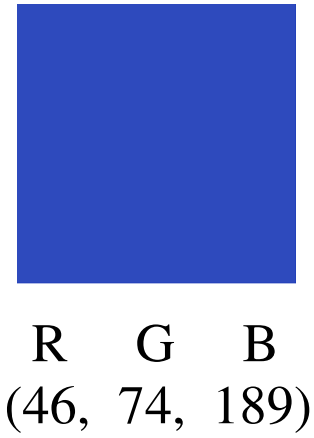

(a)
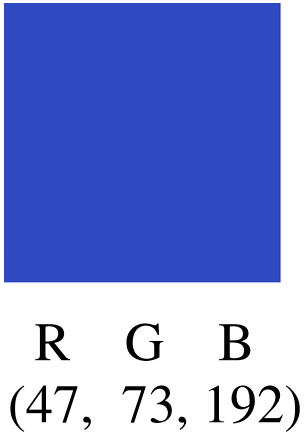

(b)

Figure 2. RGB values of an original picture element (a) and a covered version (b). 


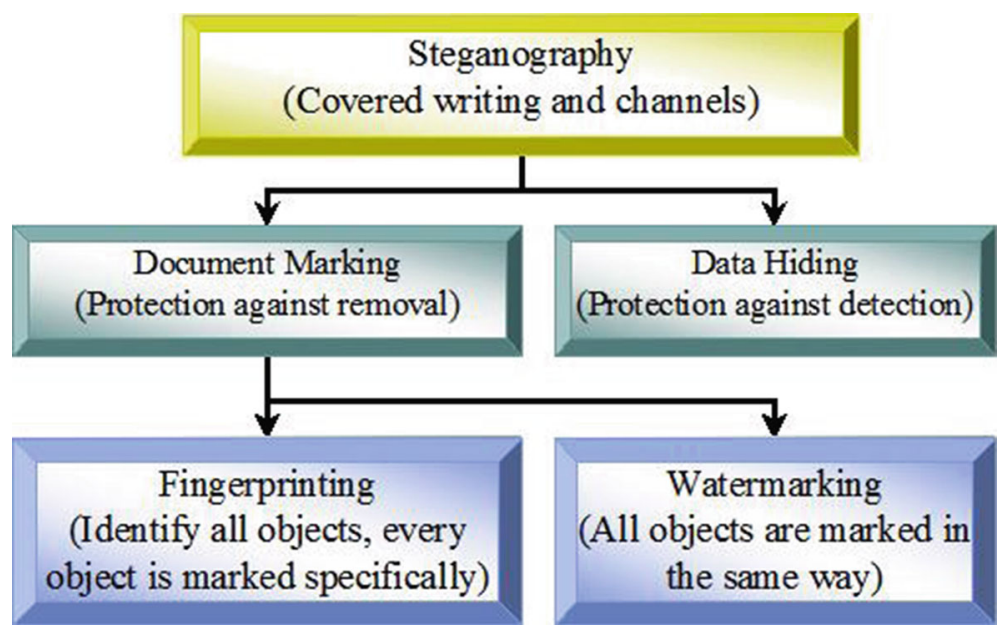

Figure 3. Information hiding disciplines.

of hiding the fact that there is a secret data (Petitcolas et al 1999). Cryptography and steganography can be combined for optimal and very dependable personal IS (Akar \& Varol 2004; Bui et al 2010).

There are a lots of methods about secret data embedding for digital images in the literature. Applications of them affect the cover image because of adding distortion or noise on the pixels. Even if the HVS is hardly able to sense these affects, it is completely diverse considering the histogram of the covered image. For instance, the HVS cannot detect dissimilarities between the digital images in figures $4 \mathrm{a}$ and $4 \mathrm{~b}$ (cover image and covered image obtained using the classical LSB data embedding technique) whereas one can readily determine the dissimilarities between the corresponding histograms given in figures $5 \mathrm{a}$ and $5 \mathrm{~b}$. The variations observed in the image histogram depicted in figure 5b is named as 'combing effect' (Yalman \& Erturk 2009).

A combing effect fundamentally points out the distorted brightness values, and can be used to recover hidden data or at least detection of hidden message existence. As it can be easily seen

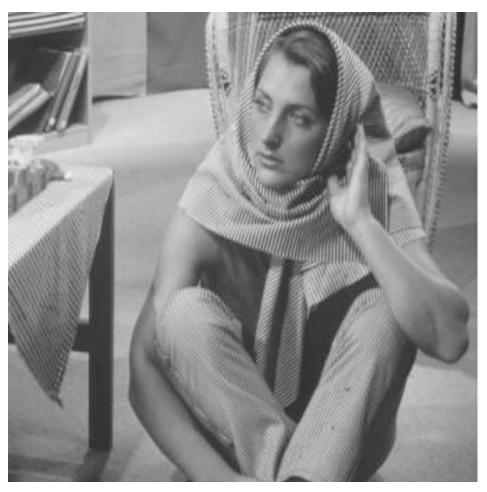

(a)

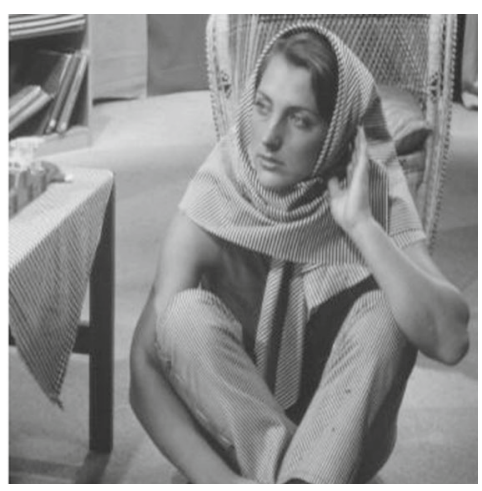

(b)

Figure 4. Test image (a) and its covered version (b). 


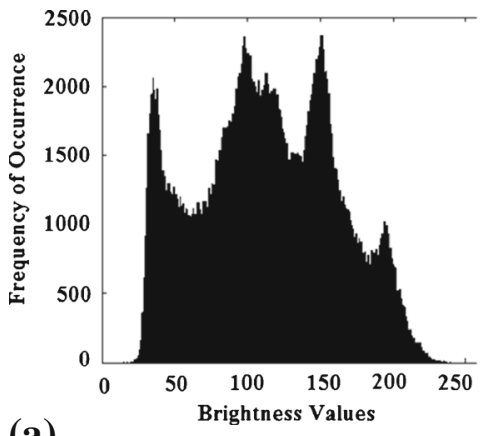

(a)

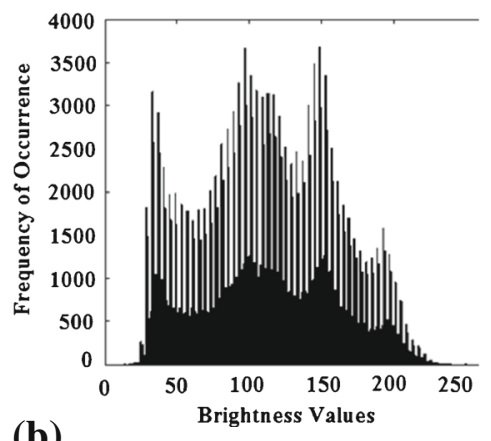

(b)

Figure 5. Histograms of the test image (a) and covered image (b).

in figures $5 \mathrm{a}$ and $5 \mathrm{~b}$, not only are the histogram views dissimilar but also the FoO of the $\mathrm{V}_{\mathrm{B}} \mathrm{S}$ are exceptionally rippled. This situation enables doing a simple assessment on a digital image histogram to check whether there is hidden data. Consequently, one could finalize that an image has been handled for a specific purpose such as transmitting a secret/personal data.

The scheme proposed in this presented study aims mainly at initiating a novel secret data embedding scheme which disguises the 'combing effect' on the covered image histogram as well as improves the PSNR and embedded data capacity of the cover image. It also significantly achieves both statistical invisibility and perceptual invisibility.

\section{Fundamentals of the proposed scheme}

The presented scheme basically operates on a digital image histogram for secret information embedding, in which neither the obtained covered image nor its modified histogram is distinctly dissimilar compared to the originals. Thus, the HVS perceives both of them as the originals, implying the fact that presented new scheme provides extremely high perceptual and statistical invisibility. The proposed approach innovatively contemplates the $\mathrm{FoO}$ of the cover image $\mathrm{V}_{\mathrm{B}} \mathrm{S}$, after that the data embedding procedure is applied based on it. The histogram of initial cover image is obtained, and the lowest and the highest $\mathrm{V}_{\mathrm{B}} \mathrm{s}$ are identified then they are called as the Lower Limit Value $\left(\mathrm{V}_{\mathrm{LL}}\right)$ and the Upper Limit Value $\left(\mathrm{V}_{\mathrm{UL}}\right)$. These two boundaries are utilized to point out where the secret data embedding procedure can be achieved (figure 6).

Flowchart of the presented scheme is depicted in figure 7. The main idea of this secret data embedding scheme and its execution procedures are clarified with an example as follows.

Assuming that the first three bits of the secret data are $(101)_{2}$ and that the cover image histogram obtained is depicted as in the figure 6 where the FoO of the 58 (i.e., $\mathrm{V}_{\mathrm{LL}}$ ), 59 and 60 (Yalman \& Erturk 2009) V V $\mathrm{B}$ s are '23', '27' and '30', respectively (table 1).

At the beginning, the proposed scheme takes that the $\mathrm{FoO}$ of the $V_{\mathrm{LL}}$ is 23 and the first secret data bit is ' 1 '. Next, it calculates the Mod2 of this FoO as ' 1 ' $(23 \operatorname{Mod} 2=1)$. Then, whether the secret data bit and the Mod2 result are the same is determined. If they are same, as in this given case (i.e., $1=1$ ), the $\mathrm{V}_{\mathrm{B}}$ stays as it is (i.e., 58) implying that it includes now the first secret data bit ' 1 '.

It then keeps on with the next coming (i.e., second) secret data bit ' 0 ' and the FoO (i.e., 27) of the next $\mathrm{V}_{\mathrm{B}}$ (i.e., 59) as the following step. As in the previous steps, the proposed scheme 


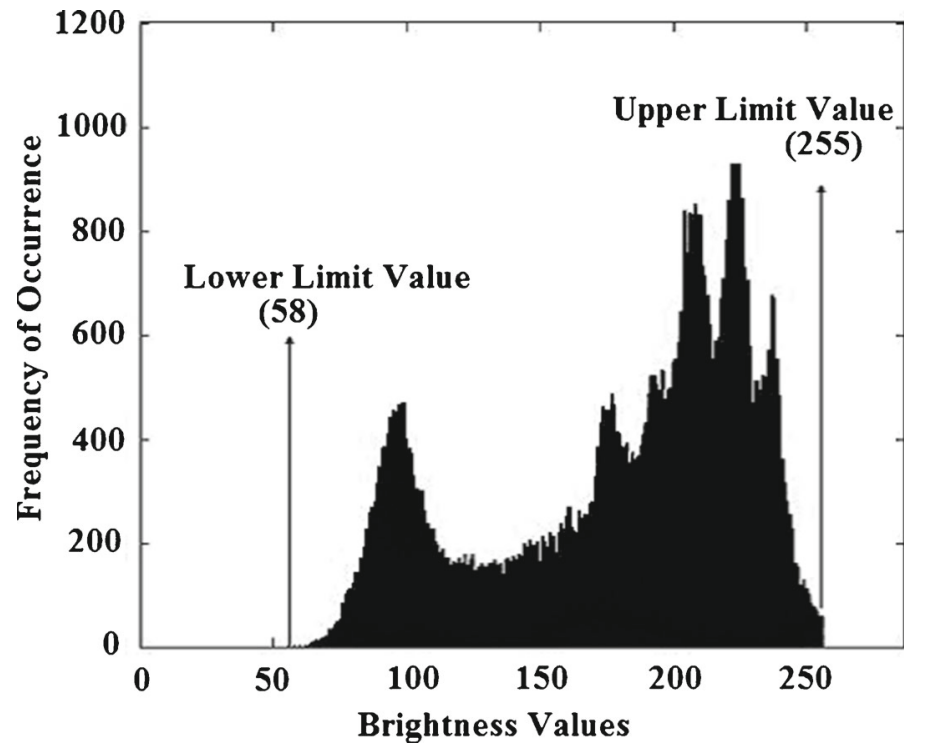

Figure 6. Identifying $\mathrm{V}_{\mathrm{LL}}$ and $\mathrm{V}_{\mathrm{UL}}$ in a histogram.

calculates the Mod2 of this FoO as ' 1 ' $(27 \operatorname{Mod} 2=1)$. Then, whether the secret data bit and the Mod2 result are the same is determined. Because they are not same (i.e., $0 \neq 1$ ), now a pixel of the cover image, whose $V_{B}$ is 59 , is revised to the next following $V_{B}$ (i.e., 60). Therefore, the resulting $\mathrm{FoO}$ of the $\mathrm{V}_{\mathrm{B}}(59)$ and $\mathrm{V}_{\mathrm{B}}(60)$ are respectively revised as '26' and '31', which ensures that the new FoO of the $\mathrm{V}_{\mathrm{B}}(59)$ includes now the second secret data bit ' 0 ' (table 2).

These given processes are performed for the next coming secret data bits and the FoO of the $\mathrm{V}_{\mathrm{B}}$ s continuously until all of the secret data bits are hidden in or the $\mathrm{V}_{\mathrm{UL}}$ is achieved indicating the cover image data hiding sources are fully utilized.

Finally, the proposed scheme takes that the third secret data bit is ' 1 ' and the FoO of the $\mathrm{V}_{\mathrm{LL}}$ is 31 . After that, it calculates the FoO Mod2 as ' 1 ' $(31 \operatorname{Mod} 2=1)$. Then, whether the secret data bit and the Mod2 result are the same is determined. If they are equal, as in this given case (i.e., $1=1$ ), the $\mathrm{V}_{\mathrm{B}}$ stays as it is (i.e., 60) meaning that it includes now the third secret data bit ' 1 ' (table 2).

The presented data embedding scheme is basically different from the classical LSB data embedding processes, where the LSB of the decimal the pixel values are altered, in that the FoO of the $\mathrm{V}_{\mathrm{B}} \mathrm{S}$ of the cover image histogram are altered to hide secret data. In other words, it is applied on decimal number domain (so it can be applied faster), but the LSB method is applied on binary number domain. It inherently overcomes the salt and pepper effect stated in some works (Ni et al 2008; Vleeschouwer et al 2003) because of the fact that the limitations of the $\mathrm{V}_{\mathrm{B}} \mathrm{s}$ of the covered image are between ' 0 ' and ' 255 '. Also, considering its deployment in 24-bit RGB colour images discretely, high data embedding capacities are made possible depending on the data size that can be hidden in each FoO (i.e., user-defined value ' $n$ ') as it is given in the equation 1 in section 3.2.

Obtaining the embedded data from the covered image is simpler than the procedures that are detailed above for secret data embedding processes (figure 8). Firstly, the covered image histogram is produced for hidden data extraction processes and the $\mathrm{V}_{\mathrm{LL}} \& \mathrm{~V}_{\mathrm{UL}}$ of the histogram are found (Yalman \& Erturk 2009). And then, Mod2 operation is carried out starting from the 


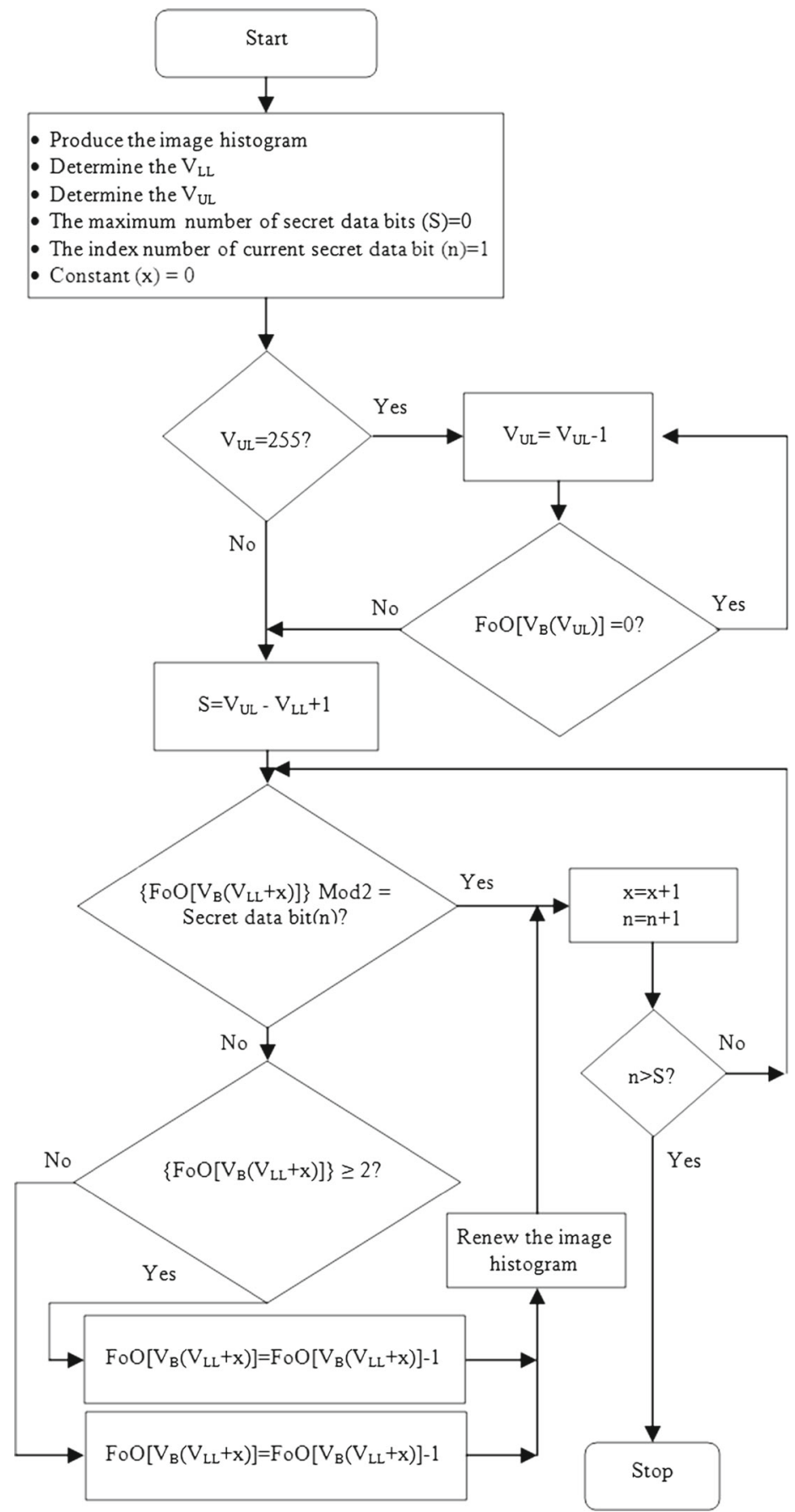

LLV: Lower Limit Value; ULV: Upper Limit Value; BV: Brightness Value; FoO: Frequency of Occurrence

Figure 7. The proposed secret data embedding scheme. 
Table 1. The first three $V_{B} s$ and their FoOs.

\begin{tabular}{llll}
\hline $\mathrm{V}_{\mathrm{B}} \mathrm{S}$ & 58 & 59 & 60 \\
FoOs of the $\mathrm{V}_{\mathrm{B}} \mathrm{s}$ in the image & 23 & 27 & 30 \\
\hline
\end{tabular}

FoO of the $\mathrm{V}_{\mathrm{LL}}$ (i.e., '23' in the given example) to $\mathrm{FoO}$ of the $\mathrm{V}_{\mathrm{UL}}$ in the histogram (Yalman et al 2012). Bearing in mind the secret data embedding case above, relatively simple procedure, which are detailed below, revealing the secret data bits (i.e., '(101) $)_{2}$ ') obtained from the covered image histogram in table 2 . As a result, the secret data bits are computed as given in figure 8 .

\subsection{Achieving higher data embedding capacity using the proposed approach}

Increasing the data embedding capacity, if needed, can be achieved by using a basic approach combining two methods, i.e., partitioning the cover image into several equal pieces and embedding two or more bits instead of a single bit of the presented scheme to the FoO of $\mathrm{V}_{\mathrm{B}} \mathrm{s}$ as detailed below. First of all, partitioning the cover image into several equal pieces is straightforward, followed by applying the developed scheme to each partition in parallel (figure 9) allows achieving higher data embedding capacities.

Secondly, as highlighted in the following example, embedding two bits to the FoO of the $\mathrm{V}_{\mathrm{B}} \mathrm{S}$ is possible, and this also allows achieving higher data embedding capacities with respect to the classical one bit for each $\mathrm{FoO}$ of the $\mathrm{V}_{\mathrm{B}} \mathrm{s}$ data embedding approach. Let's assume that the first four secret data bits are (1011) $)_{2}$ and that the cover image and its histogram obtained are depicted as in figure 6 where the $\mathrm{FoO}$ of 58, 59 and $60 \mathrm{~V}_{\mathrm{B}} \mathrm{s}$ are '23', '27' and '30', respectively (table 1).

At first, the proposed data embedding scheme takes that the first two (MSB) secret data bits are ' $(10)_{2}$ ' and the FoO of $\mathrm{V}_{\mathrm{LL}}$ is 23 . After that it calculates binary values of the FoO $(23=$ $\left.(101 \underline{11})_{2}\right)$. Then, whether the two secret data bits (i.e., $\left.(10)_{2}\right)$ and the computed last two bits are the same is determined. Because they are not same in the given example (i.e., $\left.(10)_{2} \neq(11)_{2}\right)$, now a pixel of the image, whose $\mathrm{V}_{\mathrm{B}}$ is 58 , is modified to next following $\mathrm{V}_{\mathrm{B}}$ (i.e., 59). As a result, the obtained $\mathrm{FoO}$ for the $\mathrm{V}_{\mathrm{B}}(58)$ and $\mathrm{V}_{\mathrm{B}}(59)$ are modified to '22' and '28', respectively, which means that new FoO for the $\mathrm{V}_{\mathrm{B}}(58)$ includes now the first two secret data bits ' $(10)_{2}$ ' (i.e., $\left.22=(101 \underline{10})_{2}\right)$ as given in table 3 .

It then goes on with the following second two secret data bits ' $(11)_{2}$ ' and the FoO (i.e., 28) of the next $\mathrm{V}_{\mathrm{B}}$ (i.e., 59) as following step. Similarly, the presented scheme calculates binary values of the $\mathrm{FoO}$ (i.e., $\left.28=(11100)_{2}\right)$. After that whether the secret data bits (i.e., $\left.(11)_{2}\right)$ and the calculated last two bits (i.e., $\left.(00)_{2}\right)$ are the same is determined. Because they are not same in given example (i.e., $\left.(11)_{2} \neq(00)_{2}\right)$, now three pixels of the image, whose $\mathrm{V}_{\mathrm{B}} \mathrm{S}$ are 60 , are revised to the previous $V_{B}$ (i.e., 59). Finally, the obtained new FoO for the $V_{B}(59)$ and $V_{B}(60)$ are modified to ' 31 ' and ' 27 ' respectively, which means that the new $\mathrm{FoO}$ for the $\mathrm{V}_{\mathrm{B}}(58)$ includes now the second two secret data bits ' $(11)_{2}$ ' (i.e., $\left.31=(111 \underline{11})_{2}\right)$ (table 4).

Table 2. The new values of the first three $\mathrm{V}_{\mathrm{B}} \mathrm{s}$ and their FoOs after having the given $(101)_{2}$ secret data.

\begin{tabular}{llll}
\hline $\mathrm{V}_{\mathrm{B}} \mathrm{s}$ & 58 & 59 & 60 \\
FoOs of the $\mathrm{V}_{\mathrm{B}} \mathrm{s}$ in the image & 23 & 26 & 31 \\
\hline
\end{tabular}




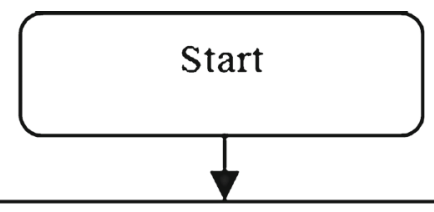

- Produce the image histogram

- Determine the $\mathrm{V}_{\mathrm{LI}}$

- Determine the VUL

- The maximum number of secret data bits $(S)=0$

- The index number of current secret data bit $(\mathrm{n})=1$

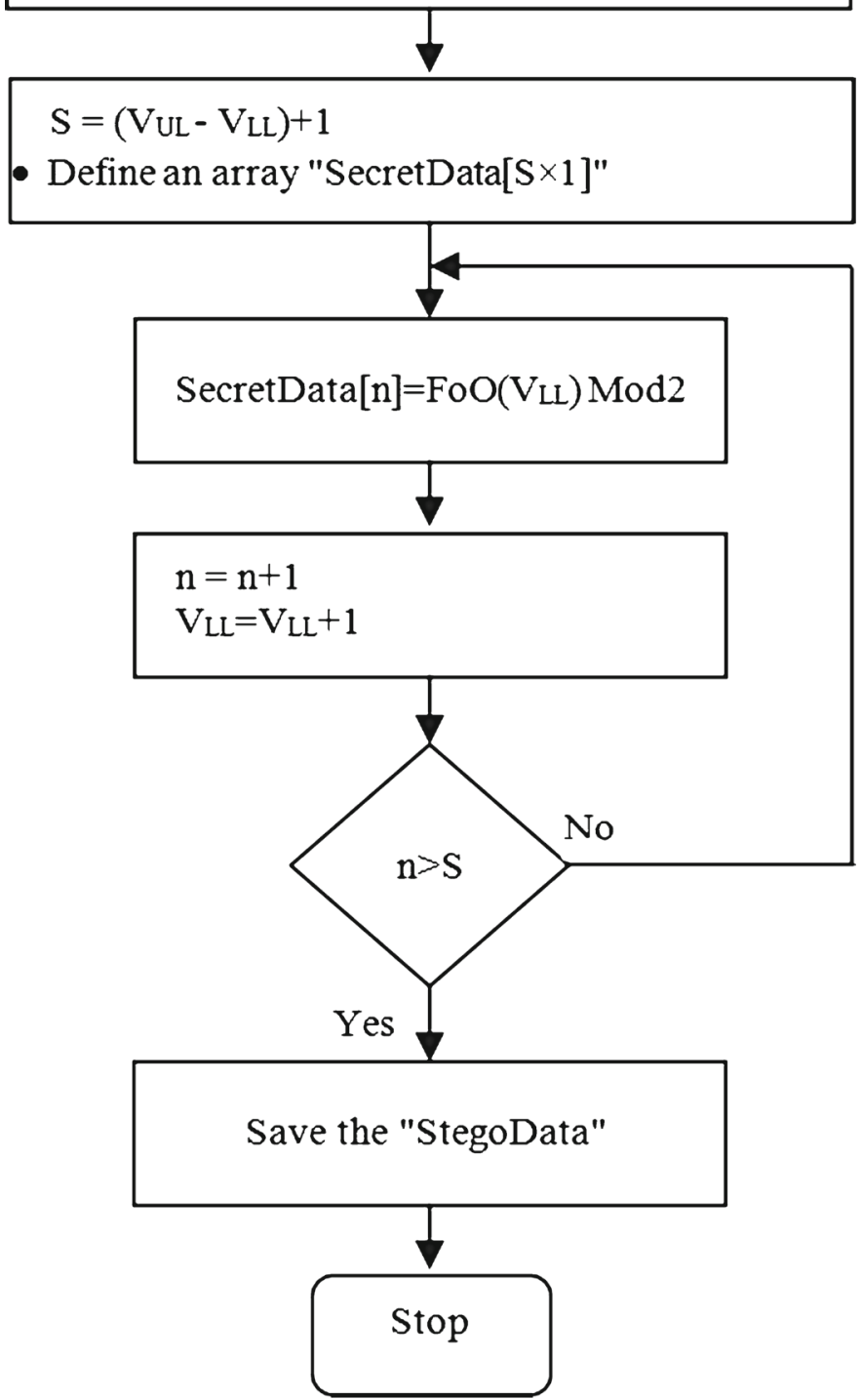

Figure 8. Flowchart for the secret data extracting. 


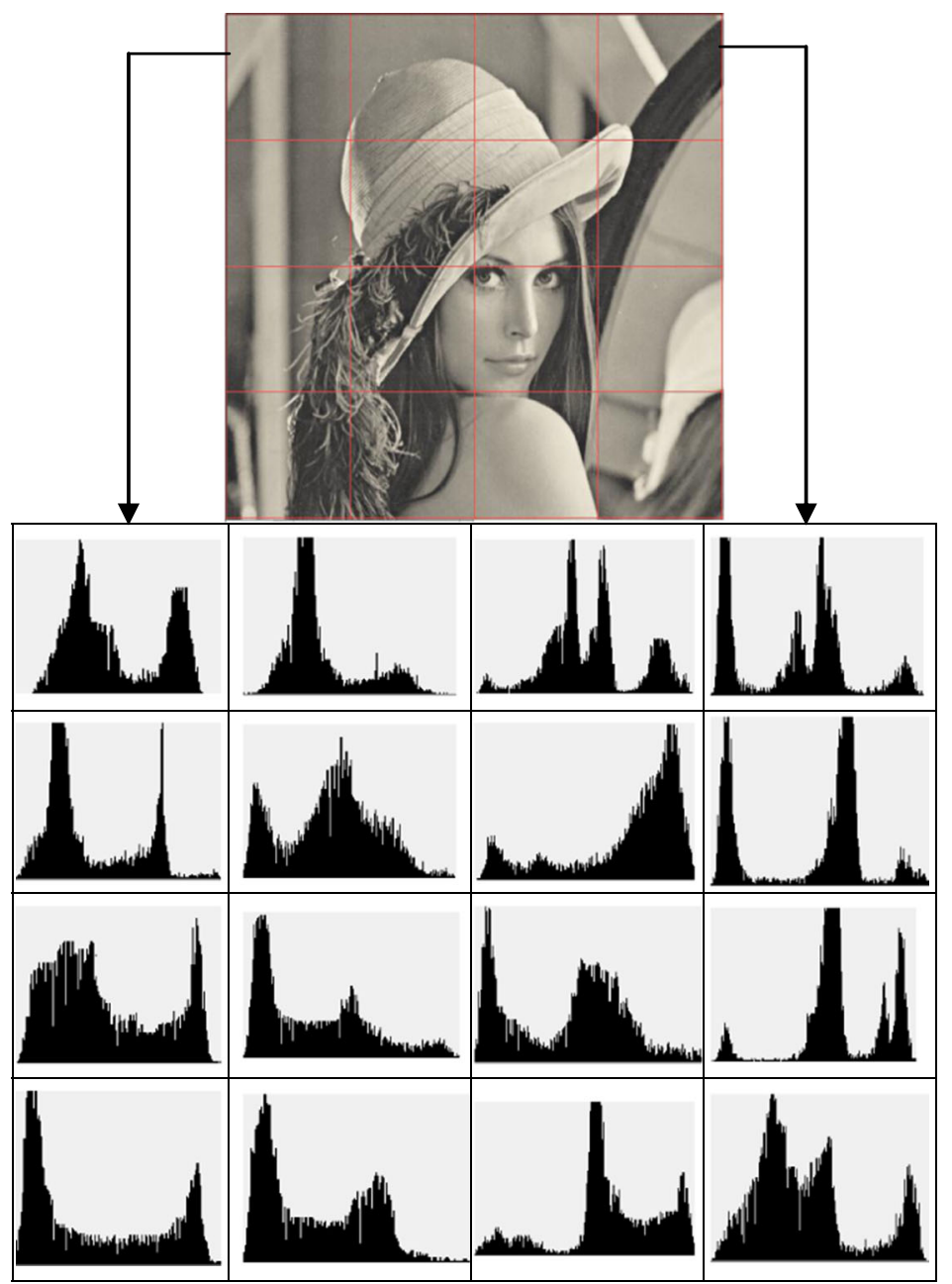

Figure 9. Partitioning the cover image for increased data embedding capacity.

Table 3. The $\mathrm{V}_{\mathrm{B}} \mathrm{s}$ and FoOs after $(10)_{2}$ data embedding procedures.

\begin{tabular}{llll}
\hline $\mathrm{V}_{\mathrm{B}} \mathrm{S}$ & 58 & 59 & 60 \\
FoOs of the $\mathrm{V}_{\mathrm{B}} \mathrm{S}$ in the image & 22 & 28 & 30 \\
\hline
\end{tabular}

Table 4. The $\mathrm{V}_{\mathrm{B}} \mathrm{s}$ and FoOs after data embedding procedures for hidden data $(1011)_{2}$.

\begin{tabular}{llll}
\hline $\mathrm{V}_{\mathrm{B}} \mathrm{s}$ & 58 & 59 & 60 \\
FoOs of the $\mathrm{V}_{\mathrm{B}} \mathrm{s}$ in the image & 22 & 31 & 27
\end{tabular}


These embedding processes are performed for all of the other following secret data bits and the FoO of the $\mathrm{V}_{\mathrm{B}}$ s continually until all of the secret data bits are embedded in or the $\mathrm{V}_{\mathrm{UL}}$ is reached implying that the cover image data embedding capacity/payload is fully used.

\subsection{Definition for the data embedding capacity}

Assume that the number of parts of the two-dimensional image (only R, G, B separately or gray) is $p$, the number of $\mathrm{V}_{\mathrm{B}} \mathrm{S}$ (for $\mathrm{FoO} \neq 0$ ) $j$, and the number of hidden data bits to be placed in each FoO is $n$, The data embedding capacity of the presented scheme is defined as follows (Yalman et al 2012):

$$
C=\left(\sum_{i=1}^{p} j_{i}\right) \times n .
$$

For example, the number of cover image parts, $\mathrm{V}_{\mathrm{B}} \mathrm{s}$ and the number of embedded data bits that can be hidden in each FoO are assumed as 6, \{170, 200, 210, 190, 185, 215 $\}$ and 2, respectively. Thus;

$$
\begin{aligned}
p & =6, \\
j_{1} & =170, j_{2}=200, j_{3}=210, j_{4}=190, j_{5}=185, j_{6}=215, \\
n & =2
\end{aligned}
$$

Therefore, the capacity $(\mathrm{C})$ can be calculated as:

$$
C=\left(\sum_{i=1}^{6} j_{i}\right) \times 2=2340 \text { bits } .
$$

\section{Evaluation of the secret data embedding scheme}

An important subject when comparing a data embedding scheme with its counterparts is the PSNR values according to the maximum data hiding capacity (it is usually called as payload). PSNR measurement is used to assess the covered image quality according to the cover image as a statistical index during experimental studies. The PSNR value enables a basic quality assessment for the covered image compared to the cover image. The higher the obtained PSNR value, the better is the data embedding process in terms of HVS perception.

At first, the Mean Square Error (MSE) is calculated using equation (3) (Sencar et al 2004; Chang et al 2003) after that the PSNR can be computed using equation (4) (Netravali \& Haskell 1995; Rabbani \& Jones 1991; Chrysochos et al 2007). Where ' $O$ ' is original image pixel value and ' $C$ ' is the covered image pixel value to be compared, and size of the image is ' $\mathrm{m} \times \mathrm{n}$ '.

$$
\begin{gathered}
M S E=\frac{1}{m \times n} \sum_{i=0}^{m-1} \sum_{j=0}^{n-1}\|O(i, j)-C(i, j)\|^{2} \\
P S N R=10 \log _{10}\left(\frac{M A X^{2}}{M S E}\right) .
\end{gathered}
$$


Table 5. The proposed data embedding scheme's basic performance.

\begin{tabular}{lccc}
\hline $\begin{array}{l}\text { Test } \\
\text { images }\end{array}$ & $\begin{array}{c}\text { Pixel deterioration } \\
\text { ratio }(\%)\end{array}$ & $\begin{array}{c}\text { Embedded } \\
\text { data (Bits) }\end{array}$ & $\begin{array}{c}\text { PSNR } \\
(\mathrm{dB})\end{array}$ \\
\hline Lena & 0.030 & 665 & 62.75 \\
Baboon & 0.010 & 747 & 59.25 \\
Peppers & 0.005 & 700 & 56.01 \\
\hline
\end{tabular}

At this point, MAX is the maximum $\mathrm{V}_{\mathrm{B}}$ number of the pixels in an image. When pixels are represented by 8 bits, MAX is defined as 255. The PSNR essentially reveals both the number of distorted bits of the pixels and the HVS perceptibility level of the changes on the cover image. Table 5 provides PSNR and data embedding capacity-based comparisons of the presented scheme's basic performance.

If the presented scheme is performed on the equally partitioned cover image pieces, the data embedding capacity (payload size) can be highly increased as the values in figure 10 prove that obviously. Especially considering the PSNR results, the presented scheme supersedes all of the other counterparts. The proposed scheme's numerical performance is shown in table 6 .

Figure 10 provides PSNR and data embedding capacity comparisons between the proposed (using both schemes described above to increase capacity) and five other classical methods (Thanuja et al 2008; Teng et al 2010; Khrisna et al 2010; Lin \& Chang 2007; Chang et al 2008) after increasing payload size.

As stated in detail above, an important issue in comparing a data embedding scheme with its counterparts is the PSNR success with respect to the payload size. In addition, the PSNR results should be as possible as greater than $30 \mathrm{~dB}$ for acceptable perceptual quality (Lee et al 2008). From this point of view, as it can be clearly seen from the plots that the method of Lin \& Chang (2007) has not only critical PSNR values varying between 33 and $30 \mathrm{~dB}$ but also its payload size

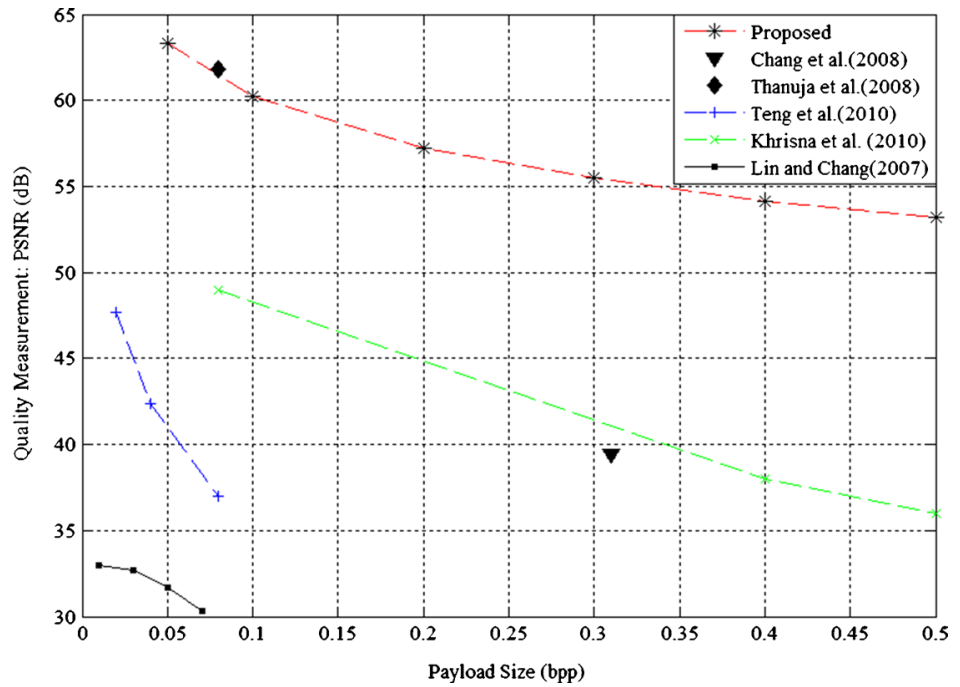

Figure 10. Comparison between the presented and other data embedding schemes for well-known Lena image $(512 \times 512)$. 
Table 6. PSNR vs. Payload size for Lena $(512 \times 512 \times 8)$.

\begin{tabular}{lcccccc}
\hline Payload (bpp) & 0.05 & 0.1 & 0.2 & 0.3 & 0.4 & 0.5 \\
PSNR (dB) & 63.29 & 60.24 & 57.23 & 55.50 & 54.17 & 53.23 \\
\hline
\end{tabular}

is about 7 times smaller than that of the proposed scheme. Although the method of Teng et al (2010) has acceptable PSNR values, its payload values are also about 6 times smaller than that of the proposed scheme and its PSNR results are diving when the payload is increased to $0.07 \mathrm{bpp}$. On the other hand, the method by Khrisna et al (2010) has almost similar payload sizes as the proposed scheme. However, its PSNR result is $13 \mathrm{~dB}$ worse than the presented scheme when the payload size is $0.1 \mathrm{bpp}$, which reaches to $18 \mathrm{~dB}$ for $0.5 \mathrm{bpp}$. This means that the PSNR results of the Khrisna et al (2010) decrease much more rapidly when the payload size is increased to 0.5 bpp. Chang et al (2008) offers a reasonable payload size but its PSNR result is noticeably smaller than that of the presented scheme, and as in the Thanuja et al (2008), the payload size cannot be increased, restricting a fair PSNR result comparison.

Experimental results of the proposed data embedding scheme on Peppers, Baboon and Lena test images are shown in figure 11. The perceptual differentiations between these cover images (i.e., a, b and c) and the corresponding covered images (i.e., d, e and f) with randomly given secret data ( $0.5 \mathrm{bpp})$ cannot be perceptually noticed by the HVS.

Figure 12 verifies the most important and beneficial outcome of the presented scheme, in which the Baboon has been used as the cover image. Figures $12 \mathrm{a}-\mathrm{c}$ are the cover image R, G and $B$ histograms, respectively. Figures $12 \mathrm{~d}-\mathrm{f}$ are the covered image histograms $(\mathrm{R}, \mathrm{G}$ and $\mathrm{B})$ acquired from the resulting covered image performing the presented scheme $(0.5 \mathrm{bpp})$ while figures $12 \mathrm{~g}-\mathrm{i}$ belong to the covered image histograms ( $\mathrm{R}, \mathrm{G}$ and $\mathrm{B})$ acquired from the resulting covered image performing a classical counterpart data embedding scheme $(0.5 \mathrm{bpp})$ (Akar \& Varol 2004).

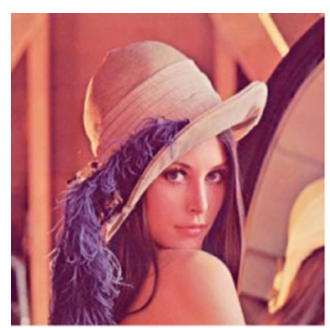

(a)

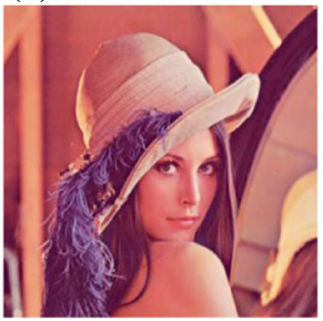

(d)

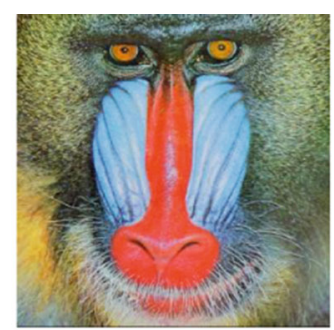

(b)

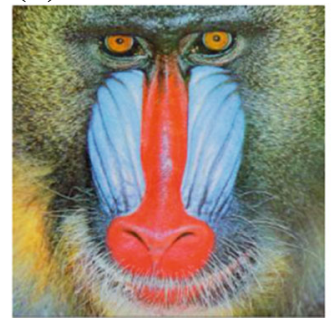

(e)

Lena

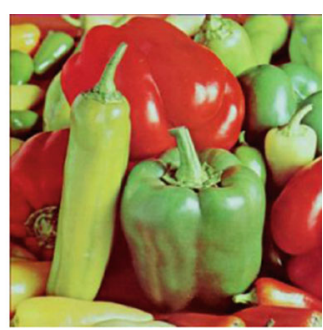

(c)

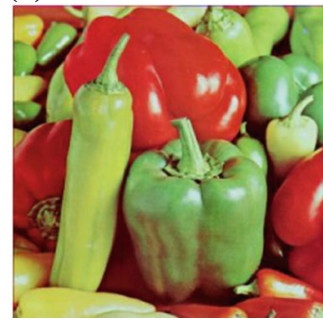

(f)

Baboon

Figure 11. Original images $(\mathbf{a}, \mathbf{b}, \mathbf{c})$ and their covered versions $(\mathbf{d}, \mathbf{e}, \mathbf{f})$ with random hidden. 


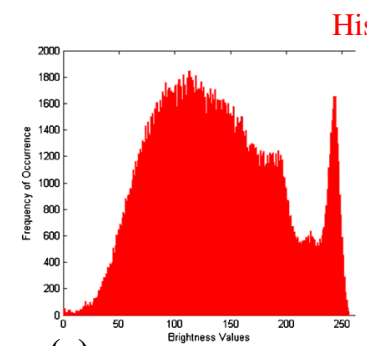

(a)

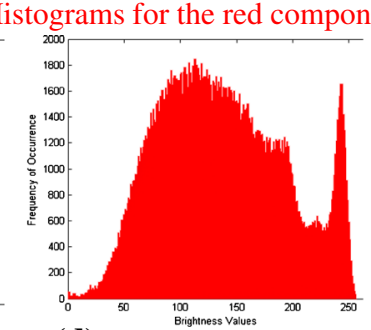

(d)

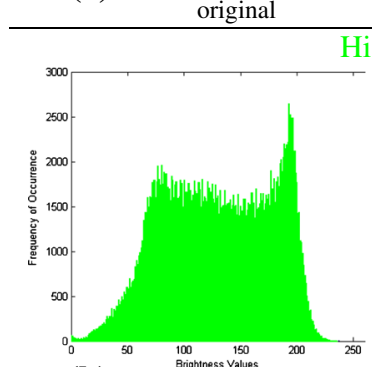

(b)

Histograms for the green component

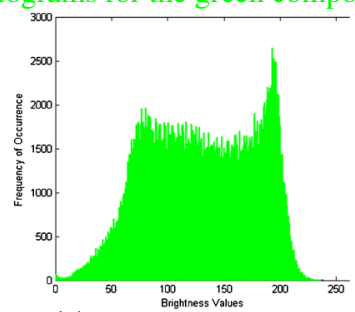

(e)

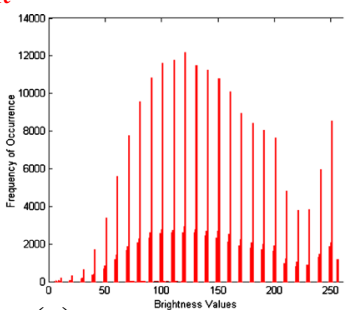

(g) classical

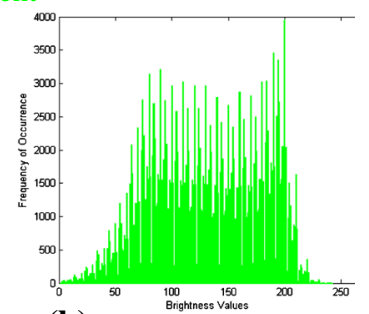

(h)

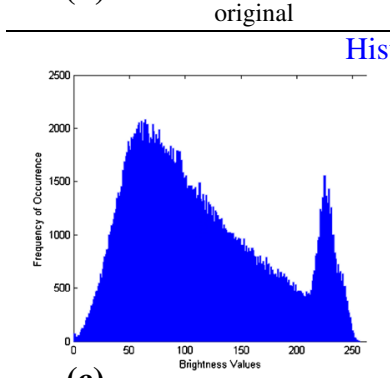

(c)

Histograms for the blue component

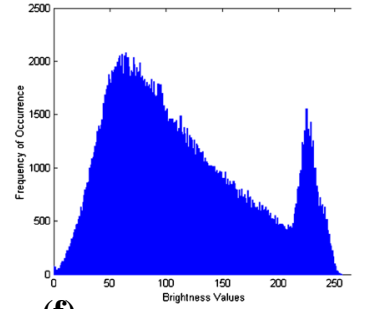

(f)

original

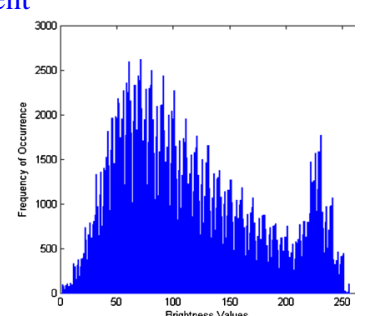

(i)

Figure 12. The original histograms (a-b-c) of the Baboon and their counterparts applying both proposed (d-e-f) data embedding scheme and the classical RGB weight-based (g-h-i) method for the covered image.

Figures $12 \mathrm{~d}-\mathrm{f}$ clearly show that there is not any modification on the image colour histograms as a result of using this presented scheme, meaning that the HVS is unable to perceive the modifications done for data embedding.

All these together indicate the facts that one cannot visually differentiate the cover and covered image histograms obtained using the proposed scheme, as well as would not be suspicious of a hidden data application in the covered image.

Apart from the points made below, using the classical data hiding algorithm's unfavourably results in highly surging and combing changes in the covered image histograms, for instance in the RGB weight-based method (e.g., figures 12g-i) (Akar \& Varol 2004).

Besides the statistical metrics, there are some perceptual assessment methods such as Mean Structural Similarity (M-SSIM) (Wang et al 2004) and Universal image Quality Index (UQI) (Wang \& Bovik 2002) to assess the proposed secret data embedding scheme. The UQI is assessed as evaluation result $(\mathrm{R})$ that ranges between [ -1 and 1] and the M-SSIM is assessed as evaluation result $(\mathrm{R})$ between $[0$ and 1$]$. The best $\mathrm{R}$ value can be 1 for both. Investigating the cover and the covered test images, UQI and M-SSIM are computed as 1, indicating the fact that the presented 
scheme guarantees completely high perceptual invisibility. Considering even the worst case scenarios changing all pixels' LSBs of the test images using the proposed scheme, the UQI and M-SSIM values can be at worse as 0.9999 and 0.9955 , respectively. This obviously reflects the superior outcome of the novel idea of modifying the FoOs of the $\mathrm{V}_{\mathrm{B}} \mathrm{s}$ for data hiding compared to classical methods.

\section{Steganalysis of the proposed scheme}

The presented data embedding scheme is examined using three well known steganalysis methods, namely Visual Steganalysis, the Raw-Quick-Pairs (RQP) and Stegdetect Tool in this section. The main purpose of a data embedding method is to hide and convey message within a cover image (Lin et al 2008). If it is possible for attackers to determine or to achieve that the cover image has secret data then the purpose of the embedding method is not achieved. These are named as detection attacks (Fridrich et al 2000). The RQP steganalysis technique is basically based on statistics of numbers of unique colours and close-colour pairs in the 24-bit colour image. A pair of colours is defined as

$$
\left|\mathrm{R}_{1}-\mathrm{R}_{2}\right| \leq 1,\left|\mathrm{G}_{1}-\mathrm{G}_{2}\right| \leq 1 \text { and }\left|\mathrm{B}_{1}-\mathrm{B}_{2}\right| \leq 1
$$

In general, when secret file is hidden into an image using LSB based method, the number of unique colours (U) rises because following proportion will increase (Fridrich et al 2000):

$$
Q=\frac{P}{\left(\begin{array}{c}
U \\
2
\end{array}\right)},
$$

where $\mathrm{P}$ is the number of close-colour pairs, and $\left(\begin{array}{l}U \\ 2\end{array}\right)$ the variation above 0.01 implies that the data hiding scheme making the covered image is validated (Sahin et al 2007). Therefore, it is readily probable to detect the presence of hidden message by adding known test bits into the covered image and observing the sum of diversity in $Q$.

Three different covered images (figures 11d-f) that are produced using our proposed secret data embedding scheme are analysed using the RQP method (table 7). It has been seen that after experiments, the difference in $Q$ values of the covered image and the covered image with embedded test bits $\left(\left|Q_{1}-Q_{2}\right|\right)$ are such high that one could not understand the entity of the hidden data in the images, validating and verifying the proposed data embedding scheme.

In addition, robustness is examined using an application named as Stegdetect for the proposed data embedding scheme (Provos \& Honeyman 2003). The output from the Stegdetect

Table 7. RQP steganalysis of the presented scheme.

\begin{tabular}{lccc}
\hline $\begin{array}{l}\text { Covered } \\
\text { image }\end{array}$ & $\begin{array}{c}Q_{1} \\
\text { (for the covered } \\
\text { image) }\end{array}$ & $\begin{array}{c}Q_{2} \\
\text { (for the covered image } \\
\text { with embedded test data) }\end{array}$ & $\left|Q_{1}-Q_{2}\right|$ \\
\hline Lena & 0.24621 & 0.22568 & 0.02053 \\
Baboon & 0.40172 & 0.38625 & 0.01547 \\
Peppers & 0.31940 & 0.30851 & 0.01089 \\
\hline
\end{tabular}


Table 8. Stegdetect steganalysis results of the presented scheme (- means that the result is negative).

\begin{tabular}{lcccccc}
\hline & \multicolumn{5}{c}{ Stegdetect results } \\
\cline { 2 - 6 } Covered image & 0.1 bpp & 0.2 bpp & 0.3 bpp & 0.4 bpp & 0.5 bpp \\
\hline Lena & - & - & - & - & - \\
Baboon & - & - & - & - & - \\
Peppers & - & - & - & - & - \\
\hline
\end{tabular}

lists either the data hiding systems found in cover images or 'negative' if no steganographic content could be sensed. Final outcomes for the three covered images given in figure 11 are all acquired as negative (table 8). Thus, this second method also verifies the use of our proposed data embedding scheme and its reliability.

Since human beings have actually interesting pattern recognition abilities and human vision monitoring ability may be successful where steganalysis techniques are insufficient (Watters et al 2005). Regarding this fact, an experimental work with 182 different participants was carried out using the three covered images produced by using our proposed data embedding scheme and their original versions (figure 11).

The experiment was performed in a computer laboratory, where the participants were presented with each target covered image and asked a question 'Is this image natural or has it been modified in some way (i.e., brightness, appearance, etc.)?' None of the participants have identified any visual inconsistency in the covered images. Secondly, the participants were given the covered and original cover images at the same time on the computer screen and are asked 'Is there any visual difference between them?'. Verifying the visual robustness of the proposed data embedding scheme, none of the participants could identify any visual differentiation between the images.

\section{Conclusions}

The presented data embedding scheme fundamentally employs the histogram modification approach. It uses the basics of image histograms and the novel idea of modifying the FoO of the $\mathrm{V}_{\mathrm{B}}$ of a digital image histogram.

Results of applying the presented scheme on the well-known test images confirm that the perceptual dissimilarities between the covered and the original images with random hidden pattern cannot be realized by the HVS. Moreover, the original image histogram is hardly different from the resulting covered image histogram as a significant output of employing the proposed scheme especially fully overcoming the combing effect. Thus, the perceptual assessment of them does not allow awareness of any data hiding application being achieved. The presented secret data embedding scheme has been also examined by using the RQP steganalysis method and the Stegdetect Tool. The results have clearly verified the robustness of the covered images against detection attacks, stating that both perceptual and statistical invisibility have been significantly achieved.

The proposed approach provides high PSNR results in addition to doubling the hidden data payload compared to its classical counterparts. The payload can also be improved both by applying a histogram widening method that expands the difference between the $\mathrm{V}_{\mathrm{UL}}$ and $\mathrm{V}_{\mathrm{LL}}$ of histogram and by partitioning the cover image into several equal pieces and then applying the presented method simultaneously. 


\section{References}

Akar F and Varol H S 2004 A new RGB weighted encoding technique for efficient information hiding in images. J. Naval Sci. Eng. 2: 21-36

Bensghir T K and Altinsoy S O 2008 A corporate restructuring proposal for the management of cyber security in Turkey, 3rd International Conference on Information Security and Cryptology 1:14-20

Bui C N, Lee H Y, Joo J C and Lee H K 2010 Secure bit-plane based steganography for secret communication. IEICE Trans. Inf. Syst. E93.D(1): 79-86

Cetin O and Ozcerit A T 2009 A new steganography algorithm based on color histograms for data embedding into raw video streams. Comput. Secur. 28: 670-682

Chang C C, Lin C C and Chen Y H 2003 Reversible data-embedding scheme using differences between original and predicted pixel values. IET Inf. Secur. 2: 35-46

Chang C C and Lin C C 2006 Reversible steganography for VQ-compressed images using side matching and relocation. IEEE Trans. Inf. Forensic Secur. 1: 493-501

Chang C C, Tai W L and Chen K N 2008 Data hiding based on histogram modification for image authentication, IEEE/IFIP Int. Conf. Embed. Ubiquit. Comput. 1: 506-511

Chen C C and Chang C C 2008 LSB-based steganography using reflected gray code. IEICE Trans. Inf. Syst. E91.D(4): 1110-1116

Chrysochos E, Fotopoulos V, Skodras A and Xenos M 2007 Reversible image watermarking based on histogram modification, $11^{\text {th }}$ Panhellenic Conf. on Informatics with International Participation 1: 93-104

Cox I J and Miller M L 2008 The first 50 years of electronic watermarking. J. Appl. Signal Process. 16: $126-132$

Fridrich J, Du R and Long M 2000 Steganalysis of LSB encoding in color images, IEEE Int. Conf. Multimed. Expo. (ICME) 3: 1279-1282

Hernandez-Castro J C, Blasco-Lopez I, Estevez-Tapiador and Ribagorda-Garnacho J M 2006 A Steganography in games: A general methodology and its application to the game of go. Comput. Secur. 25: 6471

Huang H C and Fang W C 2008 Intelligent multimedia data hiding techniques and applications, IEEE Int. Conf. Inf. Secur. Assur. 1: 477-482

Khrisna S L V, Rahim B A, Shaik F and Rajan K S 2010 Lossless embedding using pixel differences and histogram shifting technique. IEEE Recent Adv. Space Technol. Serv. Clim. Chang. (RSTSCC) 1: 213-216

Lee C C, Wu H C, Tsai C S and Chu Y P 2008 Adaptive lossless steganographic scheme with centralized difference expansion. Pattern Recog. 41: 2097-2106

Lin C N and Chang C C 2007 A best-pair-first capacity-distortion control for data hiding on vq compression domain, IEEE International Conference on $3^{\text {rd }}$ Intelligent Information Hiding and Multimedia Signal Processing 1: 509-514

Lin C Y, Chang C C and Wang Y Z 2008 Reversible steganographic method with high payload for jpeg images. IEICE Trans. Inf. Syst. E91.D(3): 836-845

Netravali A N and Haskell B G 1995 Digital pictures: representation, compression and standards. New York: Plenum Press

Ni Z, Shi Y Q, Ansari N, Su W, Sun Q and Lin W 2004 Robust lossless image data hiding. IEEE Int. Conf. Multimed. Expo. (ICME) 1: 2199-2202

Ni Z, Shi Y Q, Ansari N, Su W, Sun Q and Lin X 2008 Robust lossless image data hiding designed for semi-fragile image authentication. IEEE Trans. Circ. Syst. Video Technol. 18: 497-509

Petitcolas F A, Anderson R J and Kuhn M G 1999 Information hiding-A Survey. IEEE Spec. Issue Prot. Multimed. Content 87: 1061-1078

Provos N and Honeyman P 2003 Hide and Seek: An introduction to steganography. IEEE Secur. \& Priv. Mag.32-44

Rabbani M and Jones P W 1991 Digital image compression techniques. Washington: SPIE Optical Engineering Press 
Sahin A, Bulus E, Sakalli M T and Bulus H N 2007 The grasp of the hidden information on images with the RQP steganalysis method, IX $X^{\text {th }}$ Akademik Bilisim Conferences: 83-877 (In Turkish)

Sencar H T, Ramkumar M and Akansu A N 2004 Data hiding fundamentals and applications. New York: Elsevier Academic Press

Sevgi L 200111 September 2001 - Electronic wars, information security and national defense in changing world, Turkey: EMO special issue

Teng C Y, Shiau Y H and Chen C C 2010 A Data hiding algorithm based on histogram re-quantization, IEEE $5^{\text {th }}$ International Conference on Computer Sciences and Convergence Information Technology (ICCIT) 1: 1088-1091

Thanuja T C, Nagaraj R and Kumari M U 2008 Data hiding using increased peak histogram, IEEE Int. Workshop Data Min. Artif. Intell. (DMAI'08) 1: 44-47

Vleeschouwer C D, Delaigle J F and Macq B 2003 Circular interpretation of bijective transformations in lossless watermarking for media asset management. IEEE Trans. Multimedia 5: 97-105

Wang Z and Bovik A C 2002 A universal image quality index. IEEE Signal Proc. Lett. 9: 81-84

Wang Z, Bovik A C, Sheikh H D and Simoncelli E P 2004 Image quality assessment: from error visibility to structural similarity. IEEE Trans. Image Process. 13: 600-612

Watters P A, Martin F and Stripf H S 2005 Visual steganalysis of LSB-encoded natural images, IEEE 3rd International Conference on Information Technology and Applications 1: 746-751

Yalman Y and Erturk I 2009 A new histogram modification based robust image data hiding technique, IEEE $24^{\text {th }}$ International Symposium on Computer and Information Sciences (ISCIS'09) 1: 39-43

Yalman Y 2010 Design and implementation of a steganography method based on histogram modification for digital images, Ph.D. Thesis, Kocaeli University, Institute of Natural Science, Kocaeli (In Turkish)

Yalman Y, Akar F and Erturk I 2012 Recent advances in steganography (Chapter 4: contemporary approaches to the histogram modification based data hiding techniques), InTech Open Access Publisher 